J3eA - Vol. 3 - 2 (2004).

DOI : 10.1051/bib-j3ea:2004002

\title{
Utilisation d'un logiciel de calcul formel - Maple V - dans l'enseignement de Traitement du Signal
}

\section{T. Talbert * * (Université de Perpignan)}

Mis en ligne le 9 février 2004.

\begin{abstract}
Résumé
Cet article présente une librairie d'électronique analogique et une librairie d'électronique numérique pour Maple V (un logiciel de calcul formel). Ces deux librairies nous permettent de couvrir l'ensemble du cours de traitement du signal enseigné en licence E.E.A. Cet outil pédagogique (Maple avec les deux librairies) permet à l'étudiant de mettre en pratique sur des exemples réels les techniques de résolution apprises en cours. De par ses grandes qualités (prise en main, techniques de résolution, etc...) Maple $\mathrm{V}$ permet à l'étudiant de se focaliser uniquement sur les méthodes de résolution, tout en écartant les écueils traditionnels que sont les erreurs de signes, les résolutions d'équations différentielles, les transformées de Laplace, etc.

Mots-clés : Électronique, traitement du signal, simulation, calcul formel, Maple V.

(C) EDP Sciences, 2004.
\end{abstract}

\footnotetext{
Niveau de connaissances requis. Cours classique de traitement du signal. Aucune connaisance en informatique n'est nécessaire.

Niveau des étudiants. Deuxième cycle, licence EEA.

\begin{abstract}
* Thierry Talbert ${ }^{1}$ est un ancien élève de l'Université de Montpellier. Il a obtenu le doctorat de génie électrique en 2001 au Laboratoire d'Électrotechnique de Montpellier. Il est actuellement maître de conférences à

l'Université de Perpignan où il est en charge de l'enseignement de l'électronique et de l'électrotechnique en licence E.E.A. Son activité de recherche actuelle porte sur la modélisation - thermique et électromagnétique des composants passifs, et sur la théorie des problèmes inverses - thermique et électromagnétique - appliquée à la fiabilité des composants et systèmes.

a e-mail : talbert@univ-perp.fr

http://www.perso.club_internet.fr/ttalbert
\end{abstract}

\footnotetext{
${ }^{1}$ Laboratoire de Physique Appliquée et d'Automatique, Université de Perpignan, 52 avenue Paul Alduy, F-66860 Perpignan Cedex, France.
} 


\section{Motivations}

Il y a maintenant trois ans, il nous a été demandé d'effectuer à l'Université Montpellier II des travaux pratiques informatisés de traitement du signal en licence E.E.A. Le but de ces TP était de mettre en pratique les techniques de résolution enseignées en cours sur des exemples réels. Un logiciel de calcul numérique (Matlab...) ne pouvait convenir, puisqu'il est plus difficile (par exemple) d'effectuer des manipulations d'expressions avec ce type de logiciel. Notre choix s'est donc porté naturellement sur le logiciel de calcul formel Maple V de la société Waterloo.

\section{Pourquoi Maple V?}

Maple $\mathrm{V}$ est un langage de manipulation symbolique qui permet, grâce à une large bibliothèque de fonctions bien adaptées, de réaliser aussi bien des calculs formels que numériques. Il permet de développer, factoriser ou dériver des expressions symboliques, c'est-à-dire des formules mathématiques qui font intervenir des variables, notées par exemple $x$ ou $y$. À la différence d'un logiciel numérique, Maple résout les problèmes de manière formelle, il ne donne pas une solution approchée numérique, mais la solution exacte au problème posé. Les calculs sont effectués de manière rigoureuse et stricte, en suivant les règles de l'algèbre, sans la moindre approximation numérique. Un logiciel de calcul formel permet de manipuler tous les types d'objets mathématiques :

- les nombres ou valeurs numériques, rationnelles ou irrationnelles : $1 ; 2,1 ; \mathrm{e} ; \pi \ldots$;

- les expressions symboliques : $3 \cdot x^{3}-2 \cdot x+2 \cdot y \cdot x$,

$$
\frac{\partial^{2} U}{\partial x^{2}}+\frac{\partial^{2} U}{\partial y^{2}}-\frac{\partial^{2} U}{\partial x \partial y} \text {. }
$$

- les fonctions :

$$
(x, y) \rightarrow x^{2}-2 \cdot x \cdot y+y^{2}
$$

- les matrices :

$$
\left[\begin{array}{ll}
a & b \\
c & d
\end{array}\right]
$$

- les objets géométriques : points, segments, droites, courbes...

- etc.

Maple V comporte aussi un langage de programmation complet qui permet d'adapter les commandes Maple existantes ou d'écrire des programmes plus élaborés correspondant aux besoins de l'utilisateur. Basé sur une syntaxe proche du Pascal et du C, l'adaptation au logiciel par un étudiant ayant quelques notions de programmation ne pose aucun problème.

Ces travaux pratiques se déroulent sur 12 séances suivant 5 thèmes :

- les manipulations d'expressions : découverte de Maple V et applications sur des exercices simples (loi des noeuds, des mailles, etc.) ;

- utilisations des équations différentielles ;

- transformée de Laplace et systèmes causals ;

- le diagramme de Bode et ses applications dans les fonctions de filtrages ;

- transformée de Fourier et initiation au traitement du signal numérique (SONAR, Compact Disc Audio, etc.).

Ces travaux pratiques ont nécessité le développement de quatre librairies pour simplifier et/ou automatiser certains calculs et/ou affichages :

- une librairie d'électronique analogique (lib_electronique);

- une librairie d'électronique numérique (lib_numerique);

- une librairie d'automatique (lib_automatique);

- une librairie d'analyse numérique (lib_analyse_num). 
La librairie d'automatique ne sera pas exposée ici car elle fait l'objet d'un article spécifique « Le calcul formel avec Maple V, un complément pour l'enseignement des asservissements ». Il en est de même pour la librairie d'analyse numérique car celle-ci est en cours de développement.

Dans tout ce qui suit nous utiliserons le terme fonction Maple, pour les fonctions qui sont directement intégrées dans Maple, et le terme fonction lib_electronique ou lib_numerique, pour les fonctions qui proviennent d'une de ces deux librairies.

\section{Les librairies}

Les deux librairies que nous avons développées permettent de couvrir la totalité du cours de traitement du signal enseigné en licence E.E.A. :

- lib_electronique comprend un ensemble complet de fonctions de transfert analogiques ;

- lib_numerique comprend l'ensemble des outils nécessaires à la numérisation de signaux.

\subsection{La librairie lib_electronique}

Cette librairie comporte plus d'une trentaine de fonctions usuelles en électronique. On peut subdiviser lib_electronique en trois groupes :

- les fonctions de transfert : on trouve ici l'ensemble des fonctions de transfert du premier et du deuxième ordre, sous leurs formes générales (ex : ${ }^{\frac{k}{1+\tau_{1} \cdot p}}$ ) et composants (ex $:^{\frac{1}{1+R \cdot C \cdot p}}$ ). Nous avons ajouté quelques structures plus « exotiques » : les structures de Rauch et de Sallen Key, amplificateur opérationnel inverseur, non-inverseur, intégrateur.

- Nous avons développé de nouvelles procédures graphiques afin de pallier les carences naturelles de Maple V concernant les graphiques semi-logarithmiques. En effet, malgré le fait que l'échelle soit logarithmique, l'échantillonnage des points ne l'est pas, ce qui donne parfois des graphiques particulièrement peu esthétiques. Nous disposons donc de deux procédures graphiques Bode et Nyquist, qui comme leurs noms l'indiquent permettent de tracer automatiquement les diagrammes de Bode (module, argument) et de Nyquist d'une fonction ou d'un ensemble de fonctions de transfert.

- nous avons ajouté aussi quelques fonctions telles le calcul d'impédances en parallèle, en série, ainsi que les ponts diviseur de courant et de tension.

\subsection{La librairie lib_numerique}

Nous avons développé cette librairie comme une initiation au traitement du signal numérique, pour éviter à l'étudiant de devoir programmer l'ensemble des routines indispensables à l'étude numérique de signaux temporels continus. Elle comprend l'ensemble des fonctions nécessaires à :

- la numérisation d'un signal : largeur de porte de numérisation, fréquence d'échantillonnage variable, etc. ;

- le calcul de la transformée de Fourier du signal numérisé ;

- l'affichage automatique du signal fréquentiel ainsi calculé.

\section{Exemples}

Nous présentons succinctement deux exemples simples que nous traitons en TP :

- le filtrage analogique d'un signal bruité par un filtre passe-bas du $1^{\mathrm{er}}$ ordre, les étudiants quant à eux compléteront l'exercice en utilisant des filtres d'ordre supérieur, voire des filtres plus complexes comme les filtres en $\mathrm{T}$ ou en $\bar{\pi}$ (le sujet complet est donné dans l'annexe A);

- la numérisation d'un signal analogique (le sujet complet est donné dans l'annexe B). 


\subsection{Filtrage analogique}

On veut étudier l'influence d'un filtre du premier ordre sur un signal bruité :

- le signal à détecter possède une fréquence de $500 \mathrm{~Hz}$ et une amplitude $5 \mathrm{~V}$, il est modélisé par une sinusoïde ;

- le bruit est composé par une somme de 3 sinusoïdes de fréquences $5 \mathrm{kHz}, 12 \mathrm{kHz}$ et $1 \mathrm{MHz}$ et d'amplitudes respectives $2,2 \mathrm{~V}, 1,3 \mathrm{~V}$ et $0,5 \mathrm{~V}$.

La résolution s'effectue en quatre étapes :

1. L'écriture du signal d'entrée et de sa transformée de Laplace correspondante (Fig. 1). On crée deux cases mémoires, la première contenant le signal d'entrée $v e(t)$ et la deuxième $V e(p)$ contenant la transformée de Laplace de ve(t) calculée grâce à la fonction Maple laplace(signal, $t, p$ ).

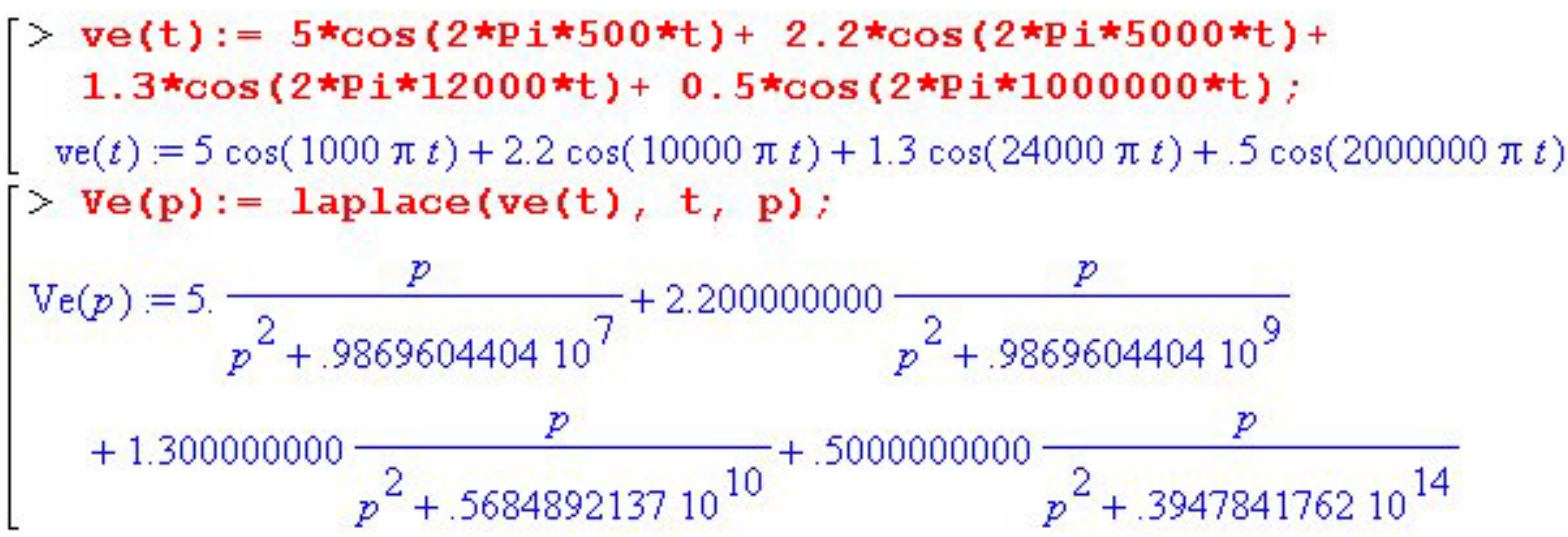

Fig. 1. Le signal d'entrée et sa transformée de Laplace.

2. L'étape numéro deux consiste à déterminer la fonction de transfert la mieux adaptée au système demandé, c'est-à-dire la constante de temps du système qui minimise le plus les parasites mais qui laisse l'amplitude du signal d'entrée la plus élevée. Le calcul sous Maple V (Fig. 2) se fait en quatre lignes : la première spécifie au logiciel que la variable recherchée est réelle, la deuxième donne l'équation à résoudre, la troisième calcule l'ensemble des solutions possibles de l'équation, la dernière sauvegarde en mémoire la valeur optimale de tau.

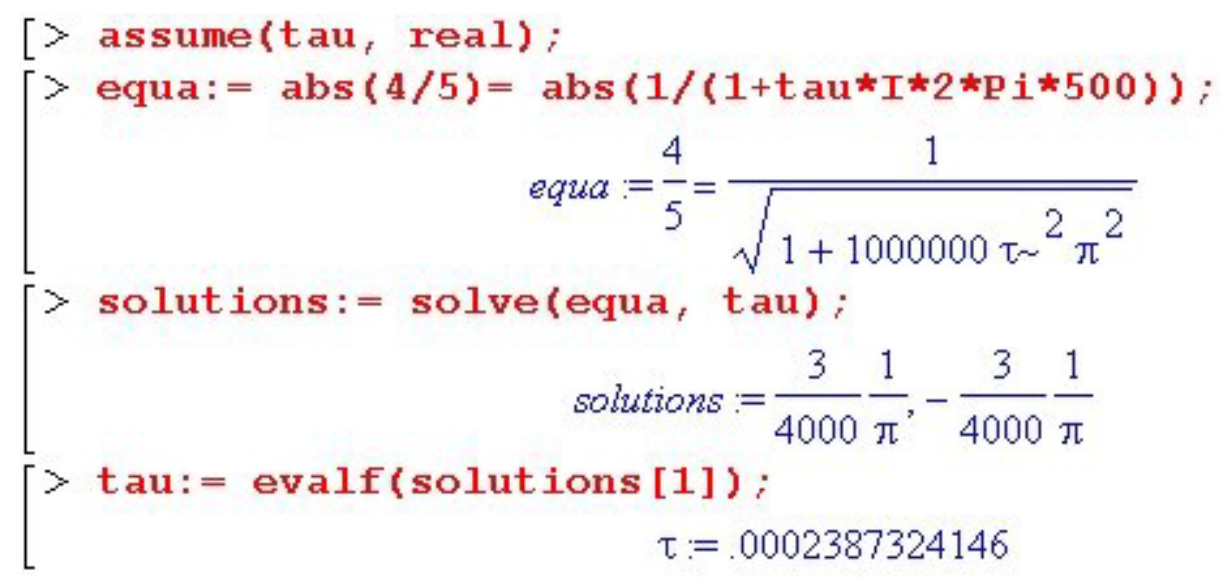

Fig. 2. Calcul du tau optimal.

3. À partir de cette solution il est alors possible de vérifier que cette fonxtion de transfert satisfait les conditions demandées (Fig. 3), c'est-à-dire qu'elle atténue le mieux possible les parasites tout en laissant 
le signal à détecter le plus important possible. Il est possible d'effectuer la vérification soit de manière traditionnelle, soit par les fonctions intégrées de lib_electronique.

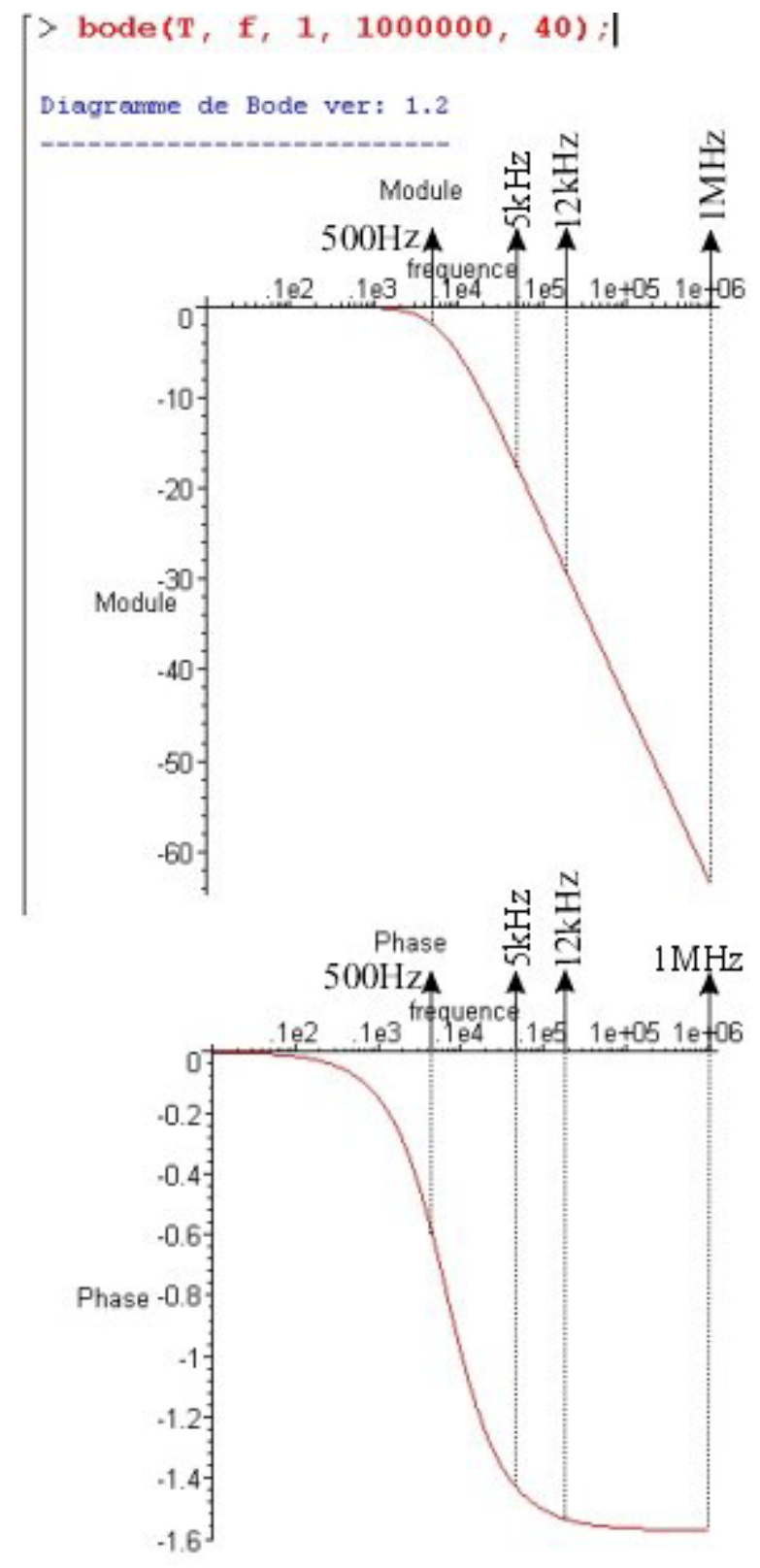

Fig. 3. Diagrammes de Bode du filtre optimal.

4. À partir de maintenant, il est parfaitement possible de reconstruire (Fig. 4) et tracer (Fig. 5) le signal de sortie après son passage dans notre filtre et d'effectuer la comparaison avec le signal bruité (Fig. 6). La résolution s'effectue à partir de la fonction Maple invlaplace(signal, $p, t)$ qui calcule la transformée inverse de Laplace du produit de la fonction de transfert du filtre par $\operatorname{Ve}(p)$. 


$$
\left[\begin{array}{rl}
> & \mathrm{vs}(\mathrm{t}):=\text { invlaplace }(\mathrm{vE}(\mathrm{p}) * \mathrm{~T}, \mathrm{p}, \mathrm{t}) ; \\
\mathrm{vs}(t) & :=-3.242428170 \mathrm{e}^{(-4188.790205 t)}+3.200000000 \cos (3141.592654 t) \\
& +2.399999999 \sin (3141.592654 t)+.03842794760 \cos (31415.92654 t) \\
& +.2882096070 \sin (31415.92654 t)+.004000000000 \cos (75398.22370 t) \\
& +.07200000000 \sin (75398.22370 t) \\
& +.222222123410^{-6} \cos \left(.628318530810^{7} t\right) \\
& +.0003333331852 \sin \left(.628318530810^{7} t\right)
\end{array}\right.
$$

Fig. 4. Calcul de l'expression analytique de la tension de sortie.

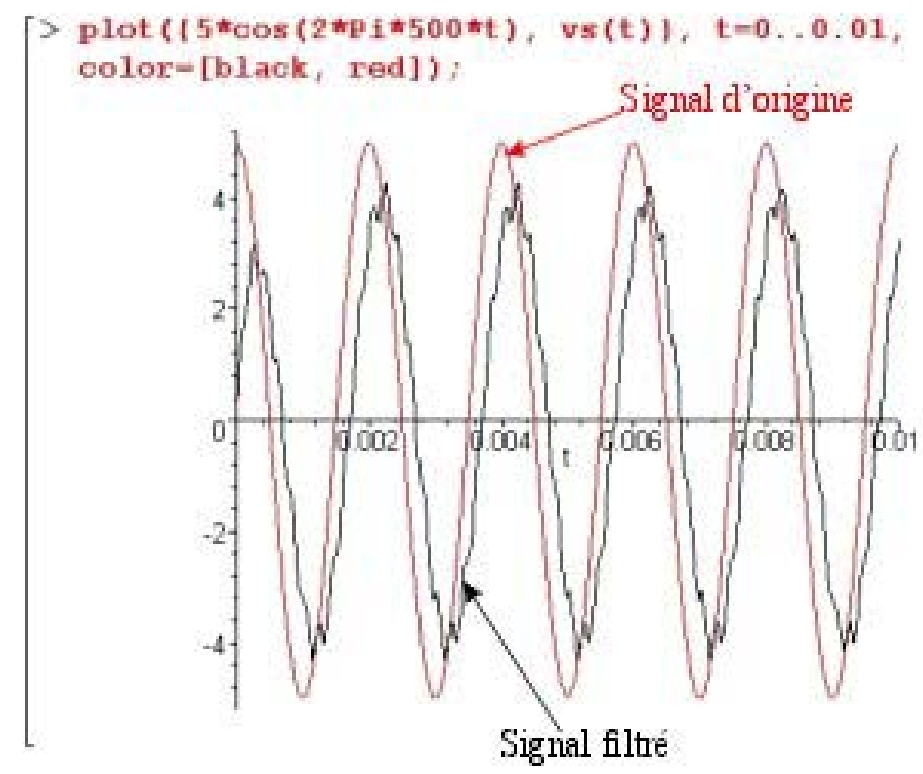

Fig. 5. Graphique de la tension de sortie et d'entrée sans les parasites.

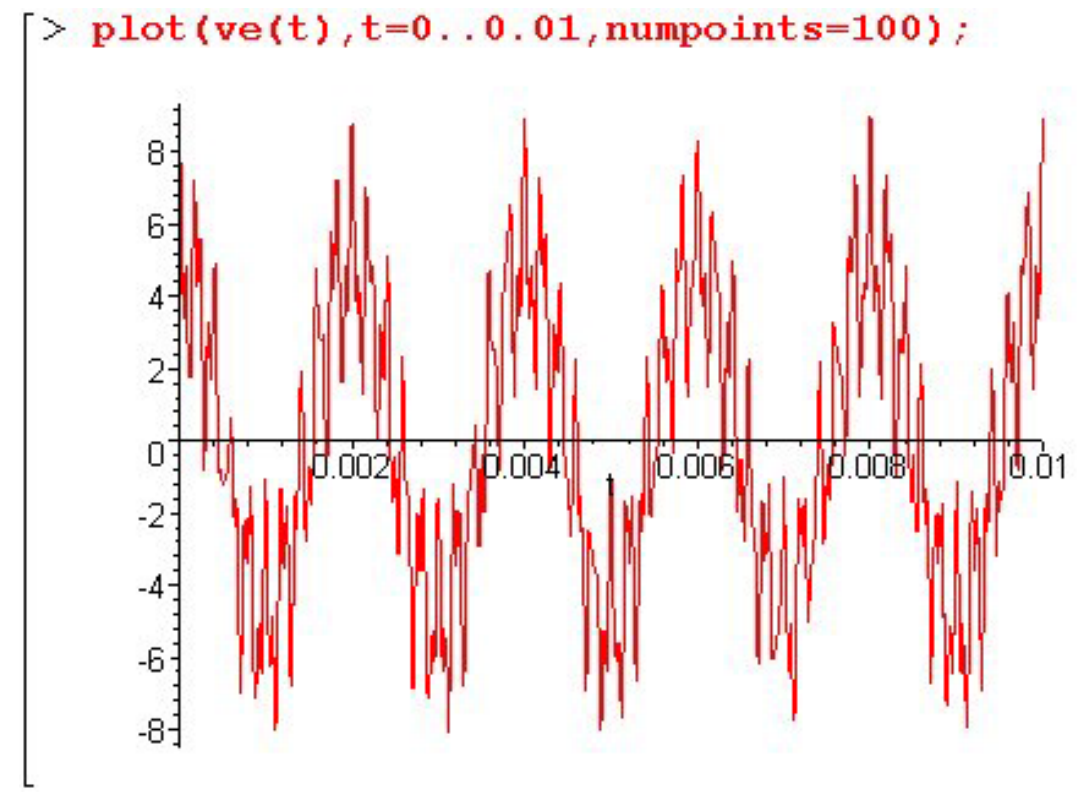

Fig. 6. Tension d'entrée avant filtrage. 
Les étudiants terminent l'exercice en proposant d'autres filtres permettant d'augmenter l'élimination des parasites. Ils peuvent soit utiliser les fonctions pré-programmées dans lib_electronique, soit les écrire eux-mêmes.

\subsection{Numérisation d'un signal}

Le deuxième exemple que nous présentons est l'étude numérique d'un signal analogique continu. Ce TP clôture l'ensemble des TP de traitement du signal de la licence. C'est un TP de transition entre l'électronique analogique et l'électronique numérique enseignée au second semestre. L'intérêt n'est pas de faire de l'électronique numérique mais d'effectuer une transition entre ces deux disciplines de l'électronique. Le but est ici de sensibiliser l'étudiant aux différents problèmes inhérents à la numérisation d'un signal qui sont entre autres :

- la fréquence d'échantillonnage ;

- la fenêtre de numérisation ;

- le nombre de points d'échantillonnage.

L'étude s'effectue complètement par la librairie lib_numerique puisque le logiciel Maple V se prête incontestablement moins bien au calcul numérique que Matlab ou Scilab. Les calculs sont faits par notre bibliothèque soit de manière semi-automatique, soit de manière automatique. Un support papier ne rendant pas compte de l'interactivité, nous réservons donc cette partie à la présentation qui se fera sur un PC sous Linux. Nous ne présentons donc que l'étude automatique dite étude rapide. Celle-ci se déroule en deux étapes :

- La définition du signal à étudier. Celui-ci est composé par quatre sinusoïdes de fréquence $100 \mathrm{~Hz}$, $1 \mathrm{kHz}, 10 \mathrm{kHz}$, et $22 \mathrm{kHz}$ (Fig. 7).

$$
\begin{aligned}
& \square \text { Fonction à échantillonner } \\
& {[>\operatorname{son}:=t->\sin (2 * \mathrm{Pi} * 100 * \mathrm{t})+\sin (2 * \mathrm{Pi} * 1000 * \mathrm{t})+} \\
& \sin (2 * \mathrm{Pi} * 10000 * \mathrm{t})+\sin (2 * \mathrm{Pi} * 22000 * \mathrm{t}) ; \\
& \text { son }:=t \rightarrow \sin (200 \pi t)+\sin (2000 \pi t)+\sin (20000 \pi t)+\sin (44000 \pi t)
\end{aligned}
$$

Fig. 7. Signal à échantillonner.

- L'appel à la fonction lib_numerique de résolution, etude freq. Cette fonction nécessite six paramètres, le nombre de points $\left(2^{10}\right)$, l'instant où débute le début de l'échantillonage $(t=0 \mathrm{~s})$, la fonction à étudier (son), les graphiques à afficher $(2=$ fréquentiel uniquement), le type d'échelle des graphiques $(0=$ échelle automatique) (Fig. 8). 


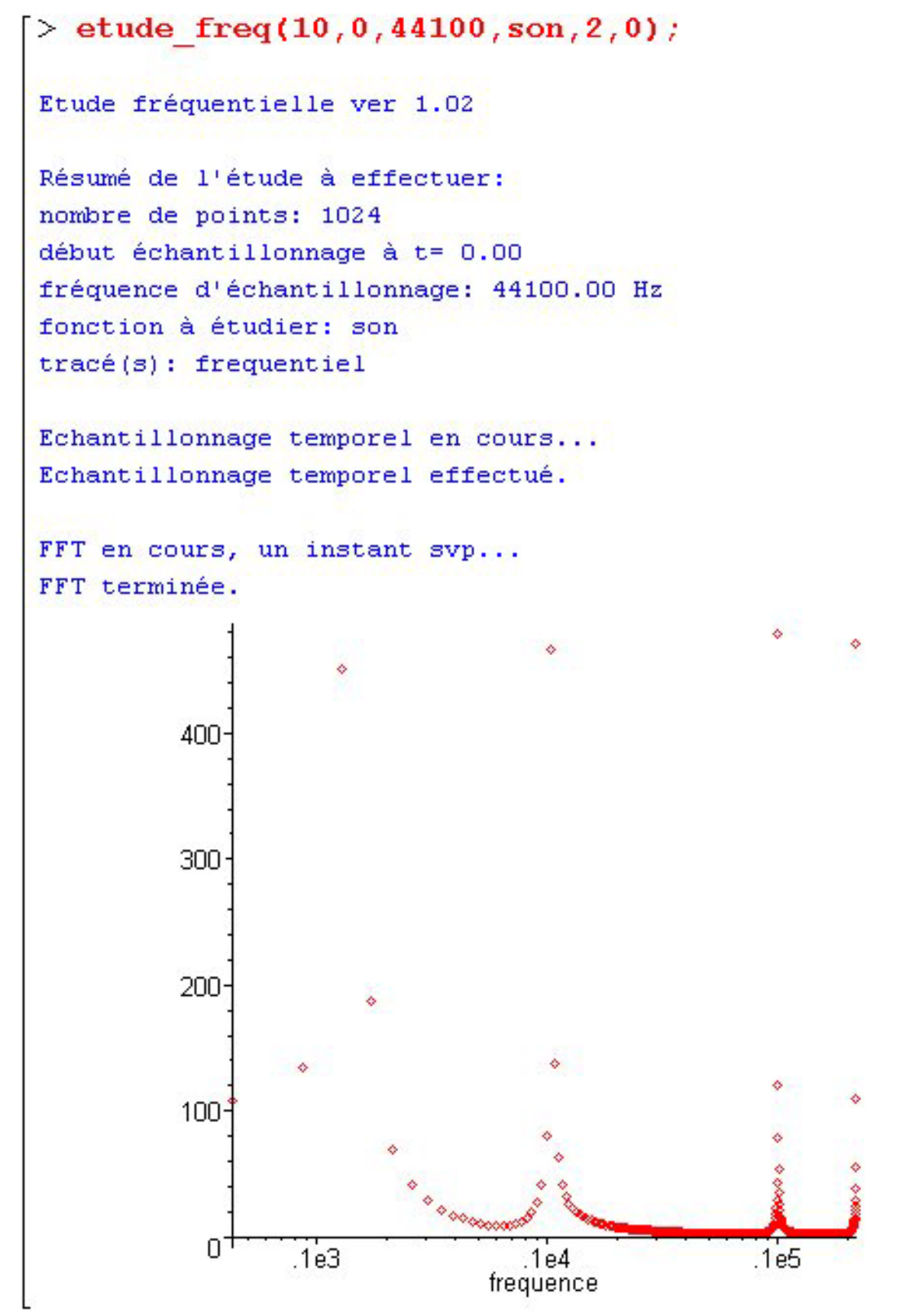

Fig. 8. Spectre du signal (fréquence échantillonnage $44,1 \mathrm{kHz}, 1024$ points).

\section{Conclusion}

Après trois ans d'expérience, les étudiants montrent un très vif intérêt pour ces travaux pratiques. Au cours des douze séances de $\mathrm{TP}$, les étudiants sont en mesure de faire le point sur leurs connaissances à partir de problèmes concrets. Ces librairies leur permettent de focaliser uniquement leur attention sur la méthodologie la plus appropriée pour résoudre un problème donné, tout en évitant les écueils traditionnels que sont les erreurs de signes, les problèmes de résolution d'équations différentielles, de transformée de Laplace, etc.

Nous travaillons actuellement à l'intégration de fonctions de filtrage numérique (FIR, etc.) ainsi que sur des fonctions de reconstitution de signaux pour la librairie lib_numerique. Ces nouvelles fonctions nous permettront de simuler une chaîne complète de traitement du signal, de la numérisation à la reconstitution en passant par le codage, puis le transport et en terminant par le filtrage. 


\section{Annexe A. Filtrage analogique}

Un étudiant en électronique (il commence à peine, il ne connaît pas encore les filtres en $\pi$, en $\mathrm{T}$, les filtres actifs, etc.) décide d'installer un filtre du premier ordre dans son montage (une cellule d'acquisition). Il a en effet découvert que des signaux sinusoïdaux (on les modélisera ainsi), hautes fréquences, d'une origine inconnue perturbent la mesure qu'il obtient.

1. Sachant qu'il doit obtenir des signaux dont la fréquence est plus faible (on les modélisera aussi par des sinusoïdes) que celles des «parasites », proposer des gabarits de filtres permettant d'éliminer ces parasites. On justifiera en traçant les diagrammes de Bode correspondants. On pourra pour cela utiliser la procédure de l'exercice précédent, si elle le permet, soit les fonctions intégrées de lib_electronique.

2. Il vient de découvrir, grâce à l'aide providentielle de son tuteur et surtout grâce à un analyseur de spectre flambant neuf, que les parasites ont les fréquences suivantes $5 \mathrm{kHz}, 12 \mathrm{kHz}$ et $1 \mathrm{MHz}$. Ce même analyseur lui a permis de déterminer les amplitudes correspondantes qui sont de 2,2 V, 1,3 V et $0,5 \mathrm{~V}$. Le signal qu'il reçoit de sa cellule d'acquisition possède une fréquence de $500 \mathrm{~Hz}$ et une amplitude de $5 \mathrm{~V}$. Déterminer une constante de temps possible pour ce filtre afin d'éliminer ces parasites, sachant qu'il doit obtenir à la sortie un signal supérieur à $4 \mathrm{~V}$. Utiliser l'exercice précédent afin de comparer (tracer) le signal d'entrée et le signal de sortie, soit en utilisant les fonctions intégrées de lib_electronique.

3. Justifier le choix d'exprimer l'ensemble des signaux par des sinusoïdes (signaux émis et parasites) et non pas sous la forme de portes (par exemple).

4. À l'aide de l'ensemble des fonctions intégrées dans le package lib_electronique, est-il possible de fabriquer des filtres possédant une meilleure atténuation (pour les parasites) que ceux proposés à la question 1) ? Tracer les gabarits possibles ainsi que la tension de sortie.

\section{Annexe B. Le CD audio}

Le schéma classique des systèmes de traitement du signal est celui-ci :

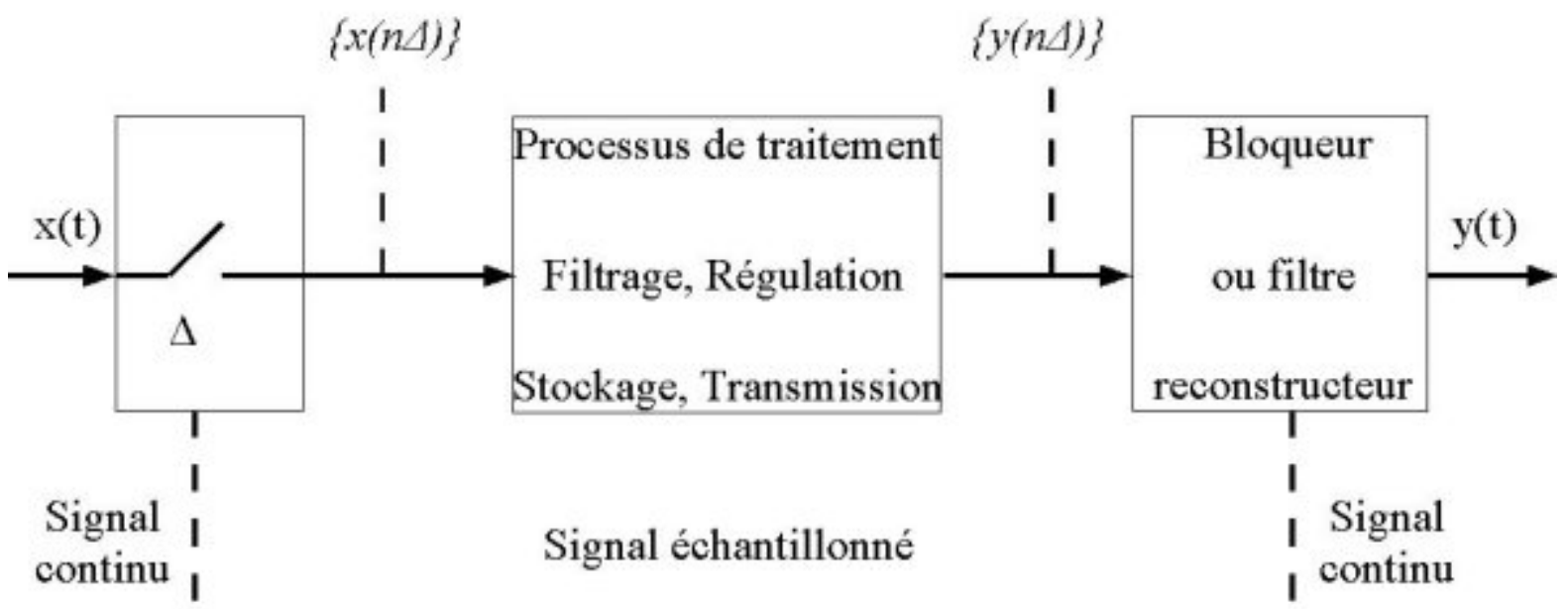

Fig. 9. Schéma classique des systèmes de traitement du signal.

Nous ne nous intéresserons ici qu'à la première partie du traitement du signal, à savoir la numérisation du signal, nous utiliserons la bibliothèque numérique [1], pour les calculs suivants. 
1. Soit la fonction suivante à numériser

- a. Calculer la transformée de Fourier de

$\operatorname{son}(t)=\sin (2 * \pi * 100 * t)+\sin (2 * \pi * 1000 * t)+\sin (2 * \pi * 10000 * t)+\sin (2 * \pi * 22000 * t)$

- b. Tracer le spectre (discret) de son $(t)$, lorsque l'on échantillonne à $44,1 \mathrm{kHz}$ et que l'on veut 1024 points.

2. Influence de la fréquence de la fréquence d'échantillonage (avec un nombre d'échantillon fixe $=1024$ points).

- a. Tracer les spectres pour les fréquences d'échantillonage suivantes : $1 \mathrm{kHz}, 22 \mathrm{kHz}$, $44 \mathrm{kHz}$ et $44,1 \mathrm{kHz}$.

- b. Interprétation des résultats.

3. Influence du nombre d'échantillon (à fréquence d'échantillonage fixe $=44,1 \mathrm{kHz}$ ).

- a. Combien de points faut-il (en théorie) pour définir une sinusoïde ?

- b. Tracer le spectre (discret) de $\operatorname{son}(t)$ avec le nombre de points minimum défini par 3-a.

- c. Tracer les spectres avec le nombre de points suivant : 2, 4, 8, 16, .. 1024.

- d. Conclusion 